\title{
Chest Pain as a Presenting Symptom for Gastric Phytobezoar
}

\author{
Ankitkumar K. Patel, MD, MPH, Sandarsh Kancherla, MD, Darren Seril, MD
}

\section{Introduction}

Chest pain is a common chief complaint of patient presentation to the emergency room. It also presents itself as one of the most challenging symptoms for clinicians to manage. The differential diagnosis for chest pain involves a multitude of organ systems. Failure to recognize potentially serious life-threatening causes such as acute ischemic heart disease, aortic dissection, tension pneumothorax, or pulmonary embolism can lead to serious morbidity and mortality. At the same time, overly conservative management of low-risk patients leads to unnecessary hospital admissions, studies and procedures. ${ }^{1}$ The following case illustrates the need to broaden the differential diagnosis for chest pain once life-threatening causes have been ruled out (Table 1).

\section{Case Presentation}

The patient is a 55 year old female with a past medical history of spinal fusion, chronic back pain, gastroesophageal reflux, and anxiety who was in her usual state of health prior to admission when she started having crushing chest pain that radiated to the left arm. The patient was resting when the pain first awoke her and was of $8 / 10$ constant intensity. The pain was associated with diaphoresis and nausea. The patient took ranitidine at first onset, but this provided no relief. The patient came into the emergency room eight hours after onset of chest pain because she was feeling fatigued and lightheaded at work.

In the emergency room the patient's pain went from $8 / 10$ to $4 / 10$ with the administration of sublingual nitroglycerin and omeprazole.

The patient's past surgical history included hysterectomy, cholecystectomy, and breast reduction. The patient's family history includes: mother with ovarian cancer, father with heart disease, and a brother with heart disease and 3 stents. The patient has a 40 pack year history of smoking cigarettes, occasional alcohol consumption, no illicit drug use and works as a cashier. The patient stated that due to the significant family history of heart disease and her significant smoking history, she had started eating a "heart healthy" diet consisting predominantly of fruits and vegetables. The patient had no known allergies. Medications on admission were omeprazole, celecoxib, naproxen, acetaminophen/hydrocodone, cyclobenzaprine, zolpidem, alprazolam, and varenicline.

On presentation, the patient was afebrile $\left(97^{\circ} \mathrm{F}\right)$ with EKG demonstrating normal sinus rhythm (72 bpm), blood pressure $100 / 58$, saturating $100 \%$ on room air with no respiratory distress noted (16 resps/min). The cardiopulmonary physical examination was within normal limits. Initial laboratory studies including a complete blood count and chemistry panel were within normal limits. A recent nuclear thallium stress test 2 months prior showed no ischemia and a LV ejection fraction of $60 \%$.
The patient was admitted to the general medicine service. Serial troponins were negative. Hemoglobin A1C level was 5.5\%. A fasting lipid panel had triglycerides of $351 \mathrm{mg} / \mathrm{dL}$, HDL of 34 $\mathrm{mg} / \mathrm{dL}$ and total cholesterol of $211 \mathrm{mg} / \mathrm{dL}$. A chest x-ray showed no active pulmonary disease and a normal cardiac silhouette. The patient underwent a nuclear thallium gated stress test which showed no exercise induced reversible myocardial perfusion defects, increased lung uptake (most likely secondary to COPD or smoking history), no evidence of regional wall motion abnormality and a calculated left ventricular ejection fraction of $58 \%$.

The patient's pain was diminished but had not been obliterated. A cardiology consultation suggested the patient be ruled out for pulmonary embolism. Though the patient did not demonstrate normal signs and symptoms of a PE, a CTA was performed which showed no signs of aortic dissection or pulmonary emboli. After a negative cardiac workup, the patient underwent endoscopy to investigate whether her symptoms were secondary to gastrointestinal pathology. The endoscopy found a medium sized phytobezoar in the stomach compatible with gastroparesis and mild antral gastritis. A gastric biopsy was taken for investigation for H. pylori. The phytobezoar was not removed.

The patient was discharged with a diagnosis of gastroparesis secondary to gastric phytobezoar with subsequent initiation of metoclopramide therapy. In addition to her previous outpatient medications, we added ezetimibe and simvastatin for hypercholesterolemia. Six weeks later, the patient reported no further episodes of chest pain.

\section{Discussion}

Traditionally, bezoars were valued as cures for disease. The name derives from the Arabic word for "antidote." ${ }^{2}$ Bezoars come in three varieties: phytobezoars, comprised of vegetable material; trichobezoars, formed from hair; and lactobezoars, composed of milk. ${ }^{3}$ Phytobezoars, the most common type of bezoar found in adults, are formed from the cellulose and lignin found in fibrous fruits and vegetables, most commonly oranges, figs, apples, persimmons, green beans, and brussel sprouts. ${ }^{4}$ Medications and supplements, such as coated aspirin, sucralfate, and vitamin C, are also known to form bezoars.

Most bezoars reside in the stomach but can be found anywhere in the gut. ${ }^{5}$ The formation of bezoars is typically associated with altered stomach physiology. Disturbances in gastric motility or gastric acid production, as well as poor mastication, are predisposing factors. Bezoars commonly arise in individuals following gastric surgeries (vagotomy, gastrectomy), and dementia or mental retardation (particularly in the case of trichobezoars). ${ }^{2}$ 
Many bezoars are asymptomatic, so the reported cases underestimate the prevalence in the population. Bezoars were found in $0.4 \%$ of patients in two large endoscopic series. ${ }^{6}$ The main risk factors for bezoar formation are abnormal mastication, vegetarian diet, ingestion of persimmons, gastric operation, diabetic gastroparesis and hypothyroidism. ${ }^{7}$ The patient described above had started a vegetarian diet.

Most cases are asymptomatic and therefore go undetected. Presenting symptoms vary depending on the site of concretion formation. Bezoars are most commonly formed in the stomach, but may also arise in the esophagus, small bowel, and rectum. Reported findings include dysphagia, abdominal pain, nausea and vomiting, early satiety, bloating, anorexia, weight loss, halitosis, constipation, and gastrointestinal bleeding. ${ }^{8}$ Phytobezoar impaction has been known to cause gastric outlet obstruction, and distal migration of gastric bezoars can cause small bowel obstruction and associated symptoms. Bezoars are capable of serving as the nidus of common bile duct stone formation. ${ }^{9}$ Physical exam may reveal a palpable abdominal mass. Imaging studies, including plain radiographs, ultrasound, and barium studies, may be useful in the diagnosis of bezoars. On CT, bezoars appear as ovoid, "mottled-appearing" or "feceslike" (gas-containing) masses. ${ }^{10}$ Endoscopy can definitively identify a concretion, while at the same time providing therapeutic options.

Treatment options include medical management, endoscopy, and surgery. Surgical approaches, laparoscopy or laparotomy, are reserved for cases where medical and endoscopic modalities fail. ${ }^{11}$ Endoscopy provides a variety of tools for the removal of bezoars, including mechanical disruption with biopsy forceps, polypectomy snares, and baskets, lithotripsy, and lasers. ${ }^{12}$ The endoscopic approach also permits the direct injection of proteolytic enzymes into the concretion. Medical management includes the use of proteolytic enzymes such as papain and cellulase, gas expanders, and mucolytics ( $\mathrm{N}$-acetylcysteine).$^{13}$ Coca-cola, administered via either endoscopic injection or lavage, has been reported effective in the management of bezoars. The sodium bicarbonate content and low $\mathrm{pH}$ of Cocacola have been cited as possible mechanisms of action. Other carbonated beverages would likely be just as effective..$^{14,15}$

The presentation of chest pain, as in the present case, is not typical of symptomatic bezoar and is not reported elsewhere in the literature. Gastric phytobezoars often present with epigastric abdominal pain, and esophageal bezoars can cause dysphagia and associated coughing and vomiting. ${ }^{16,17}$ It is well established that gastrointestinal processes, most notably gastroesophageal reflux disease (GERD) and esophageal spasm, can mimic chest pain related to ischemic heart disease. Indeed, GERD is the most common alternative explanation for the complaint of chest pain (Table 1). Esophageal bezoars are known to form in the setting of altered esophageal $\mathrm{pH}$ and GERD and there are reports of gastric bezoars impacting in the esophagus following episodes of vomiting. ${ }^{18}$ However, as mentioned above, the usual manifestation of such an esophageal obstruction is dysphagia. It is certainly possible that the bezoar in the present case was an incidental finding and was unrelated to the patient's chest pain.

On follow up 2 months later, the patient reported no more episodes of chest pain. The resolution of the chest pain symptoms with removal of the bezoar would be suggestive of an association, but would certainly not be conclusive. It is well known that chest pain due to esophageal spasm can be ameliorated by nitroglycerin, as in the present case. In addition, the patient has a history of GERD, which may provide another explanation for her symptoms. The relationship between gastric bezoars and gastroesophageal reflux has not been reported.

\begin{tabular}{|l|l|}
\hline $\begin{array}{c}\text { Table 1. Differential Diagnosis of Patients Admitted } \\
\text { to Hospital with Acute Chest Pain Ruled Out } \\
\text { for Myocardial Infarction }\end{array}$ \\
\hline Diagnosis & Percent (\%) \\
\hline Gastroesophageal reflux disease & 42 \\
\hline Ischemic heart disease & 31 \\
\hline Chest wall syndrome & 28 \\
\hline Pericarditis & 4 \\
\hline Pleuritis/pneumonia & 2 \\
\hline Pulmonary embolism & 2 \\
\hline Lung cancer & 1.5 \\
\hline Aortic aneurysm & 1 \\
\hline Aortic stenosis & 1 \\
\hline Herpes zoster & 1 \\
\hline Adapted from: Fruergaard P, et al. European Heart Journal. 1996, 17:1028. \\
\hline
\end{tabular}

\section{References}

1. Lee TH. Chest discomfort and palpitations. In: Harrison's Principles of Internal Medicine, $16^{\text {th }}$ edition. New York: McGraw Hill, 2005.

2. Zamir D, Goldblum C, Linova L, et al. Phytobezoars and trichobezoars: A 10year experience. J. Clin Gastroenterol. 2004; 38 (10): 873-876.

3. Hamilton K, Polter D. In: Sleisenger \& Fordtran's Gastrointestinal and Liver Disease: Foreign Bodies and Bezoars, $6^{\text {th }}$ Edition. Philadelphia: Saunders, 1998: 331-335.

4. Dwivedi AJ, Chahin F, et al. Gastric phytobezoar: treatment using meat tenderizer. Digestive Disease and Sciences. 2001. 46(5): 1013-1015.

5. Byrme WJ. Foreign bodies, besoars, and caustic ingestion. Gastrointestinal Endosc Clin North Amer. 1994; 4; 99-104.

6. Kadian RS, Rose JF, Mann NS. Gastric bezoars: spontaneous resolution. Am J Gastroenterol. 1978; 70:79-82.

7. Zissin R, Osadchy A, Gutman V, et al. CT findings in patients with small bowel obstruction due to phytobezoar. Emerg Radiol. 2004; 10: 197-200.

8. Brady PG, Richardson R. Gastric bezoar formation secondary to gastroparesis 